\title{
Designing artificial sodium ion reservoirs to emulate biological synapses
}

\author{
Dongshin Kim ${ }^{1}$ and Jang-Sik Lee(1)
}

\begin{abstract}
Emulating neurons/synapses in the brain is an important step to realizing highly efficient computers. This fact makes neuromorphic devices important emerging solutions to the limitations imposed by the current computing architecture. To mimic synaptic functions in the brain, it is critical to replicate ionic movements in the nervous system. It is therefore important to note that ions move easily in liquids. In this study, we demonstrate a liquid-based neuromorphic device that is capable of mimicking the movement of ions in the nervous system by controlling $\mathrm{Na}^{+}$ movement in an aqueous solution. The concentration of $\mathrm{Na}^{+}$in the solution can control the ionic conductivity of the device. The device shows short-term and long-term plasticity such as excitatory postsynaptic current, paired-pulse facilitation, potentiation, and depression, which are key properties for memorization and computation in the brain. This device has the potential to overcome the limitations of current von Neumann architecture-based computing systems and substantially advance the technology of neuromorphic computing.
\end{abstract}

\section{Introduction}

Neuromorphic devices provide efficient computing capability owing to their ability to combine computing and memory functions ${ }^{1-7}$. This architecture emulates the functions of a brain and provides a way to overcome the limitations of von Neumann architecture, which separates computing and memory functions and consequently limits the processing speed because of the need to exchange information between processors and storage sites ${ }^{8-10}$. Neuromorphic devices have been demonstrated using several types of devices, such as complementary metal-oxide semiconductors ${ }^{11,12}$, resistive switching memory ${ }^{13-20}$, phasechange memory ${ }^{21}$, spin-torque-transfer magnetic memory ${ }^{22}$, and electrochemical-based memory ${ }^{23,24}$. Electrochemicalbased devices have exhibited promising synaptic characteristics that are useful in artificial synaptic devices because the electrochemical reactions of ions in these devices can emulate the movement of ions in the nervous system ${ }^{23-29}$. While electrochemical-based neuromorphic devices have successfully mimicked various synaptic functions ${ }^{27,30}$, solid-state

\footnotetext{
Correspondence: Jang-Sik Lee (jangsik@postech.ac.kr)

${ }^{1}$ Department of Materials Science and Engineering, Pohang University of

Science and Technology (POSTECH), Pohang 37673, Korea
}

materials have limited ion diffusion to effectively emulate ionic movements in synapses ${ }^{31-33}$.

In this study, we demonstrate a neuromorphic device in which electrical signals are transported using $\mathrm{Na}^{+}$through an aqueous solution because liquids can be practical components for neuromorphic device applications ${ }^{34,35}$. Disodium terephthalate $\left(\mathrm{Na}_{2} \mathrm{TP}\right)$ is used as a $\mathrm{Na}^{+}$reservoir to regulate the concentration of $\mathrm{Na}^{+}\left(\left[\mathrm{Na}^{+}\right]\right)$in the liquid. Then, a change in $\left[\mathrm{Na}^{+}\right]$induces synaptic functions in the device. The device exhibits characteristics of biological synapses such as potentiation, depression, excitatory postsynaptic current (EPSC), paired-pulse facilitation (PPF), and spike timing-dependent plasticity (STDP). The device also has long-term plasticity (LTP) characteristics that affect memory functions in the brain ${ }^{36}$. Furthermore, because a microfluidic system can be adopted to demonstrate an integrated array of liquid-based devices, the device has the potential to be integrated using microfluidic technology.

\section{Results}

A device that controlled $\left[\mathrm{Na}^{+}\right]$was fabricated in the form of a two-terminal structure to mimic the transmission of signals between neurons and synapses (Fig. 1a). This 


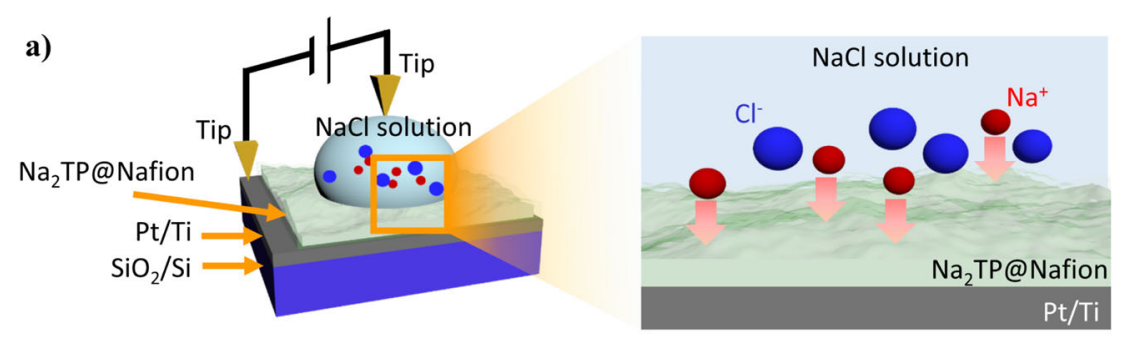

b)

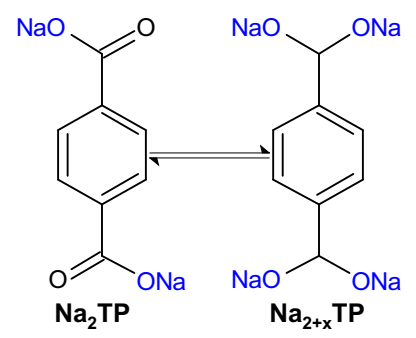

c)

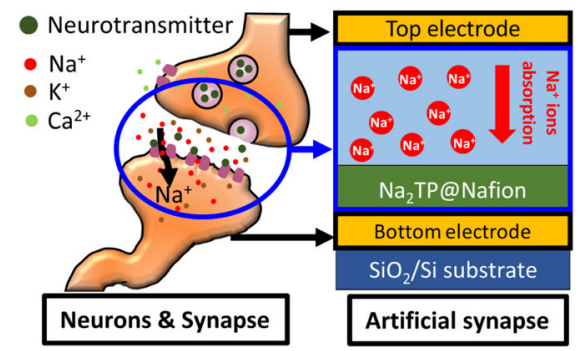

Fig. 1 Schematic device structure and biological synapse. a Left side: Structure of the device, consisting of a Pt bottom electrode, a $\mathrm{Na}_{2} \mathrm{TP} @$ Nafion layer, a $\mathrm{NaCl}$ solution, and a top electrode; Right side: Detailed schematic showing ions in the $\mathrm{NaCl}$ solution on the surface of the $\mathrm{Na}_{2} \mathrm{TP} @$ Nafion layer. $\mathbf{b}$ Chemical structure of $\mathrm{Na}_{2} \mathrm{TP}$ and insertion/deinsertion of $\mathrm{Na}^{+}$. c Comparison of a biological synapse and an artificial synapse.

artificial synapse consisted of $\mathrm{Na}_{2} \mathrm{TP}$, Nafion, a $\mathrm{NaCl}$ solution, and electrodes. $\mathrm{Na}_{2} \mathrm{TP}$ is hydrophilic; consequently, the $\mathrm{Na}_{2} \mathrm{TP}$ layer can easily absorb liquids; therefore, Nafion was used to coat $\mathrm{Na}_{2} \mathrm{TP}$ to prevent direct contact with liquid ${ }^{37-40}$. $\mathrm{Na}_{2} \mathrm{TP}$ was mixed with the Nafion solution ( $\left.\mathrm{Na}_{2} \mathrm{TP} @ N a f i o n\right)$ and deposited on the bottom electrode by spin coating. The microstructures of Nafion, $\mathrm{Na}_{2} \mathrm{TP}$, and $\mathrm{Na}_{2}$ TP@Nafion were investigated using scanning electron microscopy (SEM) (Fig. S1, Supplementary information). $\mathrm{Na}_{2} \mathrm{TP}$ has two sites that absorb $\mathrm{Na}^{+}$, and the existence of these two carbonyl groups allows for the insertion of two $\mathrm{Na}^{+}$ions (Fig. 1b) ${ }^{39-41}$. A top-electrode/ $\mathrm{NaCl}$ solution/ $\mathrm{Na}_{2} \mathrm{TP} @ \mathrm{Nafion} /$ bottom-electrode structure was used to mimic the functions of the synapse (Fig. 1c). The top and bottom electrodes acted as pre- and postsynaptic neurons, respectively, and the $\mathrm{NaCl}$ solution/ $\mathrm{Na}_{2} \mathrm{TP}$ Nafion acted as a synapse.

The device exhibited short-term plasticity (STP) and LTP characteristics of synapses. These characteristics were achieved by adjusting the frequency and amplitude of the applied pulses. STP is an important feature of computing process in the nervous system ${ }^{2,36}$. The device demonstrated STP functions, such as EPSC, PPF, and excitatory postsynaptic potential (EPSP) ${ }^{42-45}$. The conductance of the device decreased temporarily when five positive pulses $(0.5 \mathrm{~V}, 0.1 \mathrm{~s})$ were applied to the device (Fig. 2a). However, when five negative pulses $(-2 \mathrm{~V}, 0.1 \mathrm{~s})$ were applied, the current of the device increased temporarily (Fig. 2b). In both cases, the conductance of the device returned to its initial value. The EPSC characteristics of the device were measured by applying a negative pulse $(-1 \mathrm{~V}, 0.1 \mathrm{~s})$ (Fig. 2c). When voltage pulses were applied to the solution, the ions migrated; however, they drifted back to their random positions after the application of voltage pulses owing to diffusion in the solution $^{5,34}$. The device also showed EPSC characteristics with a short pulse $(-3 \mathrm{~V}, 5 \mathrm{~ms})$ (Fig. S2, Supplementary information). The device exhibited PPF, which is an increased postsynaptic response to the second pulse that arrives shortly after the first pulse (Fig. S3, Supplementary information). As the interval between the pulses decreased, the amplification of the second reaction response increased. The PPF ratio change in response to the time interval was expressed by a double-exponential function (Fig. 2d). The amplification of the responses was proportional to the frequencies of the applied pulses (Fig. S4, Supplementary information). The device also showed EPSP characteristics, which are postsynaptic potentials that allow postsynaptic neurons to transport signals $^{46}$. When a positive current pulse $(0.1 \mathrm{~mA}, 0.1 \mathrm{~s})$ was applied to the device, its potential increased temporarily and then gradually returned to the initial state (Fig. 2e). However, when pulses of positive current $(0.1 \mathrm{~mA}, 0.1 \mathrm{~s})$ were applied repeatedly, the increased potential of the device was maintained (Fig. 2f).

The LTP experienced a long-lasting change in its synaptic strength, thus suggesting the potentiation/ depression of synapses after repetitive stimulation-the result of information memorization ${ }^{47}$. When LTP was induced by the application of positive pulses to the top electrode, the device exhibited depression characteristics (Fig. 3a). The conductance of the device decreased 

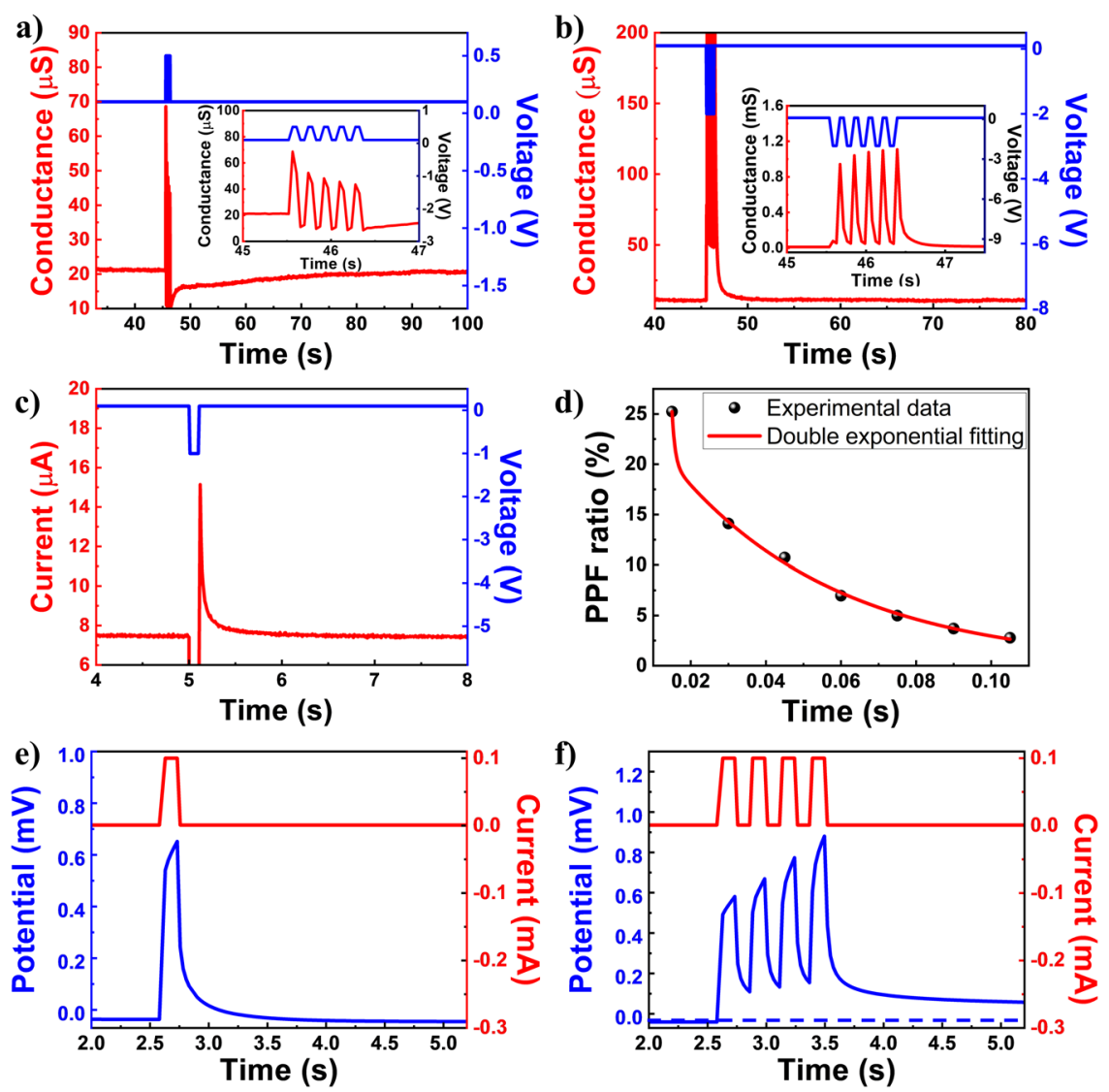

Fig. 2 Short-term synaptic plasticity characteristics of the device. a STP characteristics of the device by positive voltage stimuli. $\mathbf{b}$ STP characteristics of the device by negative voltage stimuli. c EPSC characteristics of the device. $\mathbf{d}$ PPF ratio of the device, showing that the PPF ratio decreases as the interval between stimuli increases. e EPSP characteristics of the device. $\mathbf{f}$ EPSP characteristics of the device with applications of multiple pulses.

linearly, and the device exhibited multiple different states during the depression process. To isolate the effects of ion diffusion in the solution, the conductance of the device was read $5 \mathrm{~s}$ after each stimulus was applied. To demonstrate the linear characteristics of potentiation and depression, nonidentical pulses were applied. When positive voltages were applied to the top electrode, $\mathrm{Na}^{+}$ moved to the $\mathrm{Na}_{2} \mathrm{TP} @$ Nafion layer and was absorbed in the $\mathrm{Na}_{2}$ TP. The resulting decline in $\left[\mathrm{Na}^{+}\right]$in the solution produced depression characteristics. In contrast, when negative pulses were applied to the top electrode, $\mathrm{Na}_{2} \mathrm{TP}$ released $\mathrm{Na}^{+}$into the solution, thereby increasing $\left[\mathrm{Na}^{+}\right]$ as the conductance gradually increased as a result. These processes thus demonstrated the potentiation characteristics of the device (Fig. 3b). In addition, potentiation and depression characteristics were assessed with other concentrations to validate the effect of $\left[\mathrm{Na}^{+}\right]$(Fig. S5, Supplementary information). All devices showed potentiation and depression characteristics, but the device with higher $\left[\mathrm{Na}^{+}\right]$had a larger conductance change. The device exhibited endurance characteristics of potentiation and depression operations (Fig. S6, Supplementary information). Each pulse sequence consisted of 16 positive pulses $(3 \mathrm{~V}, 0.1 \mathrm{~s})$ followed by 16 negative pulses $(-3 \mathrm{~V}, 0.1 \mathrm{~s})$. A read pulse $(0.1 \mathrm{~V}, 0.1 \mathrm{~s})$ was applied to measure conductance after each stimulation pulse. The pulse sequence was repeated more than 200 times. In addition, STDP characteristics were emulated by applying pre- and postsynaptic pulses (Fig. 3c). Pre- and postsynaptic pulses $(-3 \mathrm{~V}, 50 \mathrm{~ms})$ were applied to the top and bottom electrodes, respectively. The synaptic weight change (\%) of the device was plotted as a function of the time difference between the pre- and postsynaptic pulses. The device followed the Hebbian rule and therefore behaved similarly to biological synapses ${ }^{48,49}$. Strong stimuli resulted in LTP, which meant that the changed conductance values of the device were maintained with increasing amplitudes of the input pulses. Long-term potentiation characteristics were determined by applying 10 negative voltage pulses (Fig. 3d). When voltage pulses with amplitudes of $-3,-4$, or $-5 \mathrm{~V}$ were applied to the device, the conductance of the device was maintained at increased values. The 

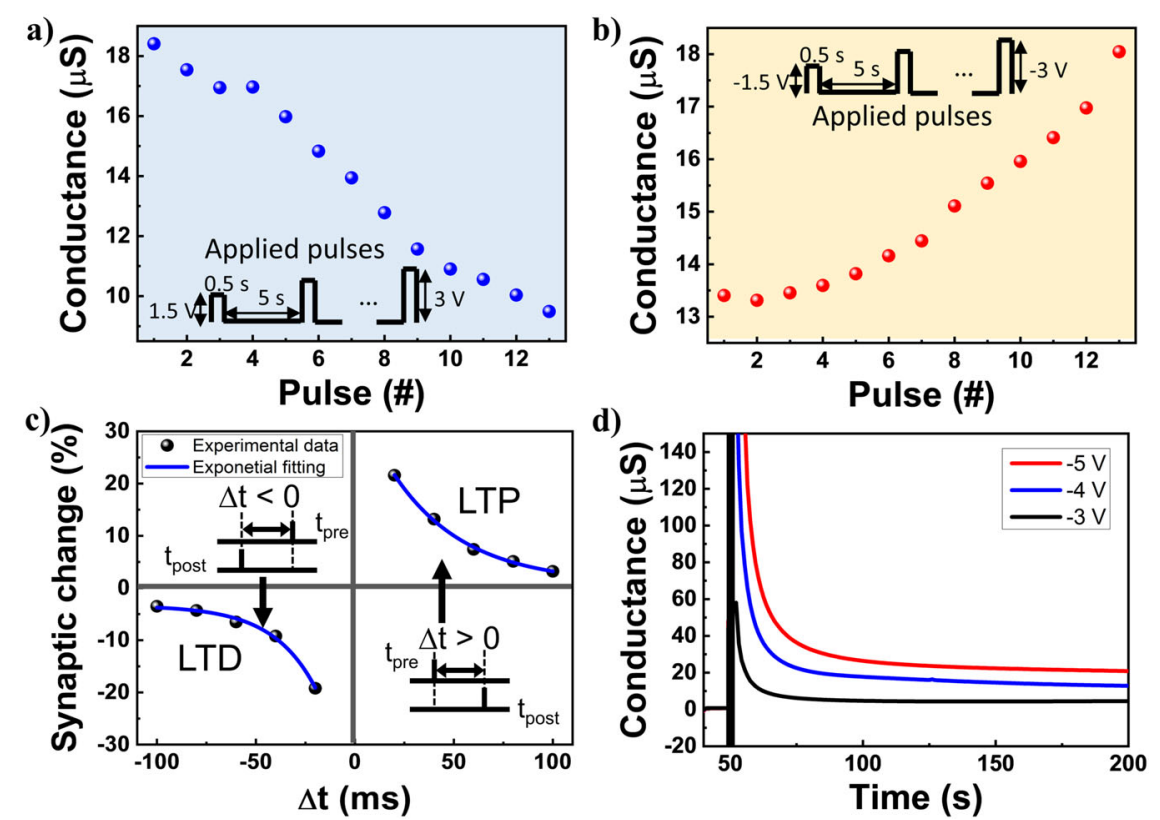

Fig. 3 Long-term synaptic plasticity characteristics of the device. a Depression characteristics of the device. $\mathbf{b}$ Potentiation characteristics of the device; nonidentical pulses were applied to the device for depression and potentiation. c STDP characteristics of the device. Pre- and postsynaptic pulses $(-3 \mathrm{~V}, 50 \mathrm{~ms})$ were applied to the top and bottom electrodes, respectively. The synaptic weight change of the device was plotted as a function of the time difference between pre- and postsynaptic pulses. $\mathbf{d}$ LTP in response to negative voltage pulses. Ten voltage pulses with amplitudes of $-3,-4$, or $-5 \mathrm{~V}$ were applied to the device. The width of the pulses was $0.1 \mathrm{~s}$.

memory states of the device were maintained for more than $600 \mathrm{~s}$ (Fig. S7, Supplementary information). However, when voltage pulses with an amplitude of $-1 \mathrm{~V}$ were applied to the device, the conductance of the device was not maintained at an increased value (Fig. S8, Supplementary information). These results imply that the device can memorize information in the long term according to the strength of the stimulus. Long-term depression characteristics were also characterized by the application of 10 positive voltage pulses (Fig. S9, Supplementary information). When voltage pulses with amplitudes of 2 , 2.5 , or $3 \mathrm{~V}$ were applied to the device, the conductance of the device was maintained at decreased values. The device showed both STP and LTP characteristics as a function of the strength of applied pulses. The emulated STP and LTP characteristics were similar to those of biological synapses, which are essential characteristics involved in the memorization and computation processes of the brain $^{50-54}$. The performance of our device was compared with that of previous artificial synapses (Table S1, Supplementary information).

\section{Discussion}

The neuromorphic characteristics of the device are due to changes in the conductance of the $\mathrm{NaCl}$ aqueous solution. The device operation is composed of four states. In the initial state, $\mathrm{Na}^{+}$and $\mathrm{Cl}^{-}$are distributed randomly in the $\mathrm{NaCl}$ solution (Fig. 4a). The $\mathrm{NaCl}$ solution and $\mathrm{Na}_{2} \mathrm{TP}$ are separated by Nafion. When positive bias pulses are applied to the device in the initial state, $\mathrm{Na}^{+}$and $\mathrm{Cl}^{-}$can be moved by bias polarity (Fig. 4b). Owing to the ion diffusion characteristics in the solution, the ionic current gradually decreases with repeated applications of stimuli. $\mathrm{Na}^{+}$cannot be absorbed in the $\mathrm{Na}_{2} \mathrm{TP}$ if the applied voltage is low; consequently, the $\left[\mathrm{Na}^{+}\right]$in the solution does not change, and the conductance of the device returns to its original state. However, when sufficient voltage is applied, $\mathrm{Na}^{+}$can pass through Nafion and react with $\mathrm{Na}_{2} \mathrm{TP} . \mathrm{Na}^{+}$is then absorbed into the $\mathrm{Na}_{2} \mathrm{TP}$, causing a decrease in $\left[\mathrm{Na}^{+}\right]$in the solution, which leads to a decrease in the conductivity of the device (Fig. 4c). When a negative voltage is applied to the device, $\mathrm{Na}^{+}$that has been absorbed in the $\mathrm{Na}_{2} \mathrm{TP}$ can be released from the $\mathrm{Na}_{2} \mathrm{TP}$ (Fig. $4 \mathrm{~d}$ ). The applied negative voltage drives an increase in the $\left[\mathrm{Na}^{+}\right]$ in the solution, causing potentiation. The number and amplitude of the bias pulses can control the $\left[\mathrm{Na}^{+}\right]$in the solution. The reactions between $\mathrm{Na}^{+}$and $\mathrm{Na}_{2} \mathrm{TP}$ were analyzed by electrochemical impedance spectroscopy (EIS). The movement and concentration of the ions can be estimated based on these EIS results ${ }^{55}$. The diameter of the semicircles in the Nyquist plot decreased when the bias voltages applied to the device increased (Fig. S10a, Supplementary information). It was inferred that the 


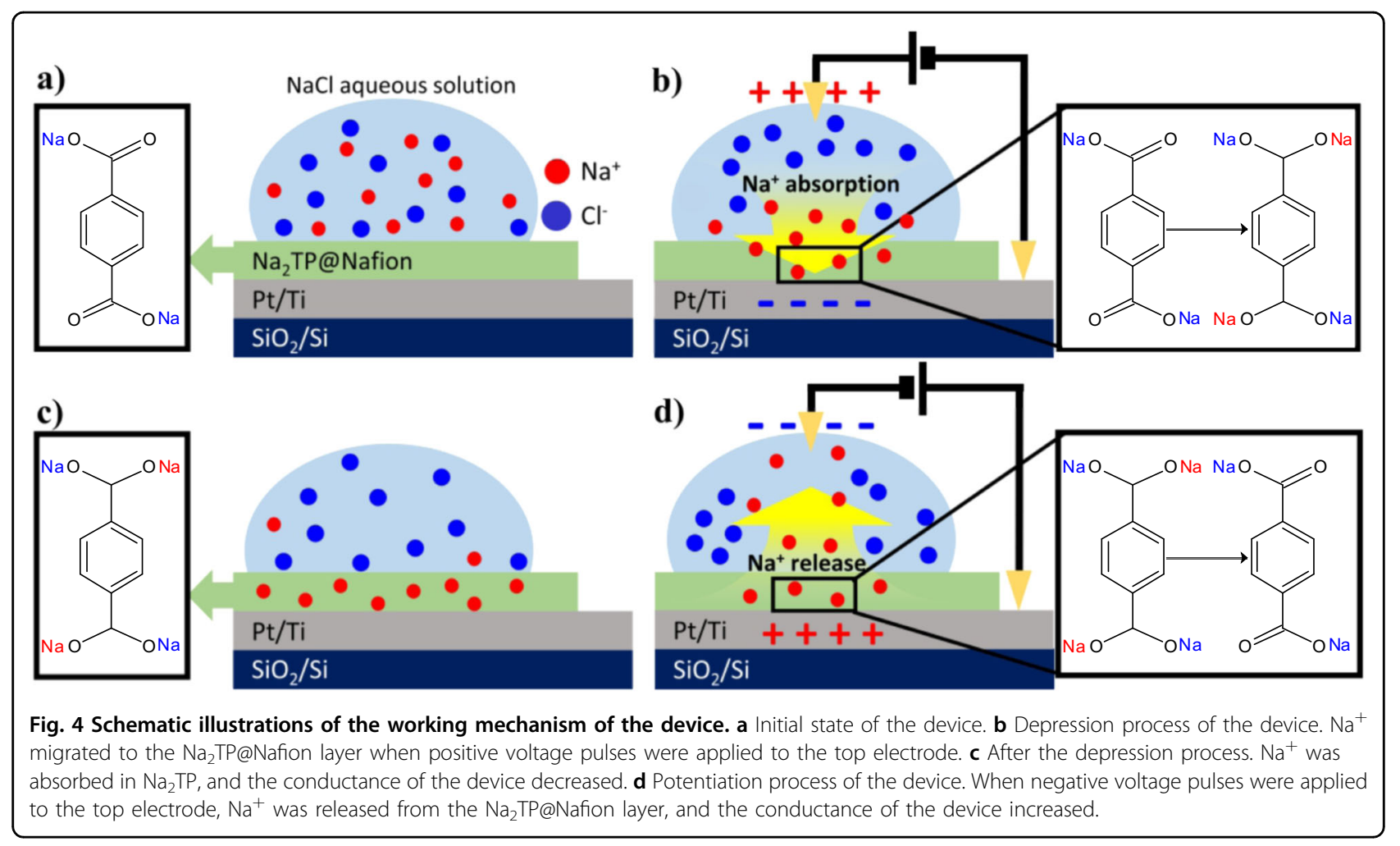

diameter of the semicircles changed owing to the reaction between $\mathrm{Na}_{2} \mathrm{TP}$ and $\mathrm{Na}^{+}$when bias voltages were applied $^{56,57}$. These characteristics were also observed in the Bode plot of impedance (Fig. S10b, Supplementary information). When the applied voltage increased, the impedance of the device decreased in the low-frequency range because of the concentration change in the solution $^{56}$.

We developed a neuromorphic device that controlled $\left[\mathrm{Na}^{+}\right]$using ion reservoirs for synaptic properties. The device, which was based on a $\mathrm{NaCl}$ solution and $\mathrm{Na}_{2} \mathrm{TP}$, exhibited the synaptic characteristics of potentiation, depression, STP, LTP, and STDP according to the applied stimuli. The signal transmission processes of the neurons were emulated using electrochemical reactions between $\mathrm{Na}^{+}$and $\mathrm{Na}_{2}$ TP@Nafion. The device used a change in $\left[\mathrm{Na}^{+}\right]$in the solution to control synaptic properties. This device utilized an aqueous solution to emulate synaptic functions. This liquid-based artificial synapse has potential applications in biocompatible devices.

\section{Methods}

\section{Materials}

Disodium terephthalate powder ( $\geq 99 \%$ purity, $\mathrm{Na}_{2} \mathrm{TP}$ ) was purchased from Alfa-Aesar (MA, USA). Nafion117 solution ( $\sim 5 \mathrm{wt} \%$ in a mixture of lower aliphatic alcohols and water) was purchased from Sigma Aldrich (MO, USA). $\mathrm{NaCl}$ powder was purchased from Samchun Chemical (Seoul,
Korea). Si wafers with 100 -nm-thick thermally grown $\mathrm{SiO}_{2}$ were used as substrates.

\section{Device fabrication}

The proposed device had a two-terminal structure. A $\mathrm{SiO}_{2} / \mathrm{Si}$ substrate with a size of $1.5 \mathrm{~cm} \times 1.5 \mathrm{~cm}$ was used. The substrate was sequentially cleaned by sonication in acetone, ethanol, and deionized water for $10 \mathrm{~min}$ each. Ti $(10 \mathrm{~nm})$ was deposited on the $\mathrm{SiO}_{2} / \mathrm{Si}$ substrate using electron-beam evaporation. Pt $(100 \mathrm{~nm})$ was then deposited using sputtering for the bottom electrode. $\mathrm{Na}_{2} \mathrm{TP} @-$ Nafion was synthesized by mixing $\mathrm{Na}_{2} \mathrm{TP}$ and Nafion at a weight ratio of 1:9. $\mathrm{Na}_{2} \mathrm{TP} @ N a f i o n$ was spin-coated on the Pt bottom electrode at $1500 \mathrm{rpm}$ for $60 \mathrm{~s}$. Then, $70 \mu \mathrm{l}$ of the $\mathrm{NaCl}$ solution was dropped on the $\mathrm{Na}_{2} \mathrm{TP} @ \mathrm{Nafion}$ layer. $\mathrm{NaCl}$ was dissolved in deionized water. Finally, the $\mathrm{Au}$ probe tip was brought in contact with the $\mathrm{NaCl}$ solution as the top electrode. The distance from the tip to the surface of $\mathrm{Na}_{2} \mathrm{TP} @$ Nafion was approximately $2 \mathrm{~mm}$.

\section{Characterization}

All electrical properties were measured under ambient conditions and at room temperature. Electrical and electrochemical characteristics were obtained using a semiconductor parameter analyzer (KEITHLEY 4200A-SCS, Tektronix Inc., OR, USA) and an electrochemical impedance analyzer (Gamry-3000, Gamry Instruments, PA, USA), respectively. During electrical measurements, the bottom 
Pt electrode was grounded, and an electrical bias was applied using the probe tip. During electrochemical measurements, the Pt electrode was used as the counter electrode, while the probe tip was used as the working electrode. The microstructures of Nafion, $\mathrm{Na}_{2} \mathrm{TP}$, and $\mathrm{Na}_{2} \mathrm{TP} @ N a f i o n$ were investigated using field-emission SEM (JSM 7800, JEOL Ltd., Tokyo, Japan).

\section{Acknowledgements}

This work was supported by the National Research Foundation of Korea (NRF2019R1A2C2084114 and 2016M3D1A1027665). Additionally, this work was supported by the Industrial Strategic Technology Development Program (20003968) funded by the Ministry of Trade, Industry \& Energy (MOTIE, Korea). In addition, this work was partially supported by the Brain Korea 21 PLUS project (Center for Creative Industrial Materials).

\section{Author contributions}

J.S.L. conceived and directed the research. J.S.L. and D.K. designed and planned the experiment. D.K. performed the experiment and acquired the data. D.K. and J.S.L. wrote the manuscript.

\section{Conflict of interest}

The authors declare that they have no conflict of interest.

\section{Publisher's note}

Springer Nature remains neutral with regard to jurisdictional claims in published maps and institutional affiliations.

Supplementary information is available for this paper at https://doi.org/ 10.1038/s41427-020-00243-2

Received: 2 April 2020 Revised: 24 June 2020 Accepted: 3 July 2020. Published online: 18 September 2020

\section{References}

1. Prezioso, M. et al. Training and operation of an integrated neuromorphic network based on metal-oxide memristors. Nature 521, 61-64 (2015).

2. Abbott, L. F. \& Regehr, W. G. Synaptic computation. Nature 431, 796-803 (2004).

3. Navarrete, A., van Schaik, C. P. \& Isler, K. Energetics and the evolution of human brain size. Nature 480, 91-93 (2011).

4. López, J. C. A fresh look at paired-pulse facilitation. Nat. Rev. Neurosci. 2, 307 (2001).

5. Xu, W., Min, S.-Y., Hwang, H. \& Lee, T.-W. Organic core-sheath nanowire artificial synapses with femtojoule energy consumption. Sci. Adv. 2, e1501326 (2016).

6. Wang, Y. et al. Emerging perovskite materials for high density data storage and artificial synapses. J. Mater. Chem. C. 6, 1600-1617 (2018).

7. Saxena, V., Wu, X., Srivastava, I. \& Zhu, K. Towards neuromorphic learning machines using emerging memory devices with brain-like energy efficiency. J. Low Power Electron. Appl. 8, 34 (2018).

8. Kim, Y. et al. A bioinspired flexible organic artificial afferent nerve. Science $\mathbf{3 6 0}$, 998-1003 (2018).

9. Shi, J., Ha, S. D., Zhou, Y., Schoofs, F. \& Ramanathan, S. A correlated nickelate synaptic transistor. Nat. Commun. 4, 2676 (2013).

10. Wang, $Y$. et al. Photonic synapses based on inorganic perovskite quantum dots for neuromorphic computing. Adv. Mater. 30, 1802883 (2018).

11. Yang, J. J., Strukov, D. B. \& Stewart, D. R. Memristive devices for computing. Nat. Nanotechnol. 8, 13-24 (2013).

12. Nazari, M. H., Mazhab-Jafari, H., Leng, L., Guenther, A. \& Genov, R. CMOS neurotransmitter microarray: 96-channel integrated potentiostat with on-die microsensors. IEEE Trans. Biomed. Circuits Syst. 7, 338-348 (2013).

13. Pan, F., Gao, S., Chen, C., Song, C. \& Zeng, F. Recent progress in resistive random access memories: Materials, switching mechanisms, and performance. Mater. Sci. Eng. R 83, 1-59 (2014).
14. Yin, J. et al. Adaptive crystallite kinetics in homogenous bilayer oxide memristor for emulating diverse synaptic plasticity. Adv. Funct. Mater. 28, 1706927 (2018).

15. Zhou, L. et al. Biological spiking synapse constructed from solution processed bimetal core-shell nanoparticle based composites. Small 14, 1800288 (2018).

16. Choi, S. et al. SiGe epitaxial memory for neuromorphic computing with reproducible high performance based on engineered dislocations. Nat. Mater. 17, 335-340 (2018).

17. Ohno, T. et al. Short-term plasticity and long-term potentiation mimicked in single inorganic synapses. Nat. Mater. 10, 591-595 (2011).

18. Wang, Z. et al. Memristors with diffusive dynamics as synaptic emulators for neuromorphic computing. Nat. Mater. 16, 101-108 (2017).

19. Kumar, S., Strachan, J. P. \& Williams, R. S. Chaotic dynamics in nanoscale $\mathrm{NbO}_{2}$ Mott memristors for analogue computing. Nature 548, 318-321 (2017).

20. Ding, G. L. et al. 2D Metal-organic framework nanosheets with timedependent and multilevel memristive switching. Adv. Funct. Mater. 29, 1806637 (2019).

21. Enyilmaz, S. B. et al. Brain-like associative learning using a nanoscale nonvolatile phase change synaptic device array. Front. Neurosci. 8, 205 (2014).

22. Torrejon, J. et al. Neuromorphic computing with nanoscale spintronic oscillators. Nature 547, 428-431 (2017).

23. van de Burgt, Y. et al. A non-volatile organic electrochemical device as a lowvoltage artificial synapse for neuromorphic computing. Nat. Mater. 16, 414-418 (2017).

24. Yang, J. J. \& Xia, Q. Organic electronics: battery-like artificial synapses. Nat. Mater. 16, 396-397 (2017).

25. Fu, Y. M. et al. Hodgkin-Huxley artificial synaptic membrane based on protonic/ electronic hybrid neuromorphic transistors. Adv. Biosyst. 2, 1700198 (2018).

26. Gkoupidenis, P., Schaefer, N., Garlan, B. \& Malliaras, G. G. Neuromorphic functions in PEDOT:PSS organic electrochemical transistors. Adv. Mater. 27, 7176-7180 (2015)

27. Lai, Q. et al. lonic/electronic hybrid materials integrated in a synaptic transistor with signal processing and learning functions. Adv. Mater. 22, 2448-2453 (2010).

28. Rivnay, J. et al. Organic electrochemical transistors. Nat. Rev. Mater. 3, 17086 (2018).

29. Steriade, M., Nunez, A. \& Amzica, F. A novel slow $(<1 \mathrm{~Hz})$ oscillation of neocortical neurons in vivo: depolarizing and hyperpolarizing components. J. Neurosci. 13, 3252-3265 (1993).

30. Wexler, E. M. \& Stanton, P. K. Priming of homosynaptic long-term depression in hippocampus by previous synaptic activity. Neuroreport $\mathbf{4}$, 591-594 (1993).

31. Koneshan, S., Rasaiah, J. C., Lynden-Bell, R. M. \& Lee, S. H. Solvent structure, dynamics, and ion mobility in aqueous solutions at $25^{\circ} \mathrm{C}$. J. Phys. Chem. B 102, 4193-4204 (1998).

32. Kushmerick, M. J. \& Podolsky, R. J. Ionic mobility in muscle cells. Science $\mathbf{1 6 6}$ 1297-1298 (1969).

33. Messerschmidt, B., Hsieh, C. H., Mclntyre, B. L. \& HoudeWalter, S. N. Ionic mobility in an ion exchanged silver-sodium boroaluminosilicate glass for micro-optics applications. J. Non-Crystalline Solids 217, 264-271 (1997).

34. Kim, D. \& Lee, J-S. Liquid-based memory and artificial synapse. Nanoscale $\mathbf{1 1}$ 9726-9732 (2019)

35. Levi, T. \& Fujii, T. Microfluidic neurons, a new way in neuromorphic engineering? Micromachines 7, 146 (2016)

36. Blitz, D. M., Foster, K. A. \& Regehr, W. G. Short-term synaptic plasticity: a comparison of two synapses. Nat. Rev. Neurosci. 5, 630-640 (2004).

37. Ellingboe, J. L. \& Runnels, J. H. Solubilities of disodium terephthalate in aqueous solutions of sodium carbonate and sodium bicarbonate. J. Chem. Eng. Data 11, 185-187 (1966).

38. Cao, C. Y., Wang, H. B., Liu, W. W., Liao, X. Z. \& Li, L. Nafion membranes as electrolyte and separator for sodium-ion battery. Int. J. Hydrog. Energ. 39, 16110-16115 (2014)

39. Sk, M. A. \& Manzhos, S. Exploring the sodium storage mechanism in disodium terephthalate as anode for organic battery using density-functional theory calculations. J. Power Sources 324, 572-581 (2016).

40. Park, Y. et al. Sodium terephthalate as an organic anode material for sodium ion batteries. Adv. Mater. 24, 3562-3567 (2012).

41. Zhao, L. et al. Disodium terephthalate $\left(\mathrm{Na}_{2} \mathrm{C}_{8} \mathrm{H}_{4} \mathrm{O}_{4}\right)$ as high performance anode material for low-cost room-temperature sodium-ion battery. Adv. Energy Mater. 2, 962-965 (2012). 
42. Sah, P., Hestrin, S. \& Nicoll, R. A. Properties of excitatory postsynaptic currents recorded in vitro from rat hippocampal interneurones. J. Physiol. 430, 605-616 (1990).

43. Schulz, P. E., Cook, E. P. \& Johnston, D. Changes in paired-pulse facilitation suggest presynaptic involvement in long-term potentiation. J. Neurosci. 14, 5325-5337 (1994)

44. Debanne, D., Guerineau, N. C., Gahwiler, B. H. \& Thompson, S. M. Paired-pulse facilitation and depression at unitary synapses in rat hippocampus: quantal fluctuation affects subsequent release. J. Physiol. 491, 163-176 (1996).

45. Malenka, R. C. Postsynaptic factors control the duration of synaptic enhancement in area CA1 of the hippocampus. Neuron 6, 53-60 (1991).

46. Luo, C. H. \& Rudy, Y. A dynamic model of the cardiac ventricular action potential. I. Simulations of ionic currents and concentration changes. Circ. Res. 74, 1071-1096 (1994).

47. Bliss, T. V. P. \& Collingridge, G. L. A synaptic model of memory: long-term potentiation in the hippocampus. Nature 361, 31-39 (1993).

48. Caporale, N. \& Dan, Y. Spike timing-dependent plasticity: a Hebbian learning rule. Annu. Rev. Neurosci. 31, 25-46 (2008).

49. Bi, G.-Q. \& Poo, M.-M. Synaptic modifications in cultured hippocampal neurons: dependence on spike timing, synaptic strength, and postsynaptic cell type. J. Neurosci. 18, 10464-10472 (1998).
50. Kaeser, P. S. \& Sudhof, T. C. RIM function in short- and long-term synaptic plasticity. Biochem. Soc. Trans. 33, 1345-1349 (2005).

51. Lisman, J., Cooper, K., Sehgal, M. \& Silva, A. J. Memory formation depends on both synapse-specific modifications of synaptic strength and cell-specific increases in excitability. Nat. Neurosci. 21, 309-314 (2018).

52. Chang, T., Jo, S. H. \& Lu, W. Short-term memory to long-term memory transition in a nanoscale memristor. ACS Nano 5, 7669-7676 (2011).

53. Kim, M. K. \& Lee, J.-S. Short-term plasticity and long-term potentiation in artificial biosynapses with diffusive dynamics. ACS Nano 12, 1680-1687 (2018)

54. Park, Y. \& Lee, J.-S. Artificial synapses with short- and long-term memory for spiking neural networks based on renewable materials. ACS Nano 11 8962-8969 (2017).

55. Barsoukov, E., et al. in Impedance Spectroscopy: Theory, Experiment, and Applications 2nd edn (eds Barsoukov E. \& Macdonald J. R.) (John Wiley \& Sons, Inc., New Jersey, NJ, USA, 2018).

56. Lück, J. \& Latz, A. The electrochemical double layer and its impedance behavior in lithium-ion batteries. Phys. Chem. Chem. Phys. 21, 14753-14765 (2019).

57. Cueto-Gómez, L. F., Garcia-Gómez, N. A., Mosqueda, H. A. \& Sánchez, E. M. Electrochemical study of $\mathrm{TiO}_{2}$ modified with silver nanoparticles upon $\mathrm{CO}_{2}$ reduction. J. Appl. Electrochem. 44, 675-682 (2014). 